\title{
INSPECTION OF PROFILED FRP COMPOSITE STRUCTURES BY MICROWAVE NDE
}

\author{
Asha Gokul, Srinivas Kuchipudi and J. Dhanasekaran \\ Scientists, Directorate of NDE \\ Dr. APJ Abdul Kalam Missile Complex, Advanced Systems Laboratory, DRDO, \\ Hyderabad-500058, Telangana, India
}

\begin{abstract}
Fiber reinforced polymer (FRP) composites are employed in various applications of aerospace and defence industry. FRP composites are preferred as major structural parts due to their high stiffness strength and light weight.Non-destructive evaluation (NDE) plays an important role in assessing the quality and health monitoring of FRP composite structures during their manufacturing and in-service period.Different NDE techniques, such as ultrasonics, thermography, X-ray radiography, etc are employed for evaluating the quality of the composite structures.Microwave non-destructive evaluation $(M W N D E)$ is an emerging NDE technique for characterizing and inspecting dielectric structures. Microwave NDE finds application in the areas of dielectric material characterization, determining thickness variation, defect detection and bond quality inspection.Inspection of profiled FRP composite structures by near-field reflection microwave NDE technique is presented in this paper. Application of Microwave NDE for bond quality inspection of FRP composite structures and thickness variation of composite structures is discussed. Results of inspected profiled composite structures by swept frequency reflection microwave NDE technique in the frequency range of X-band and $K u$-band respectively are presented.
\end{abstract}

\section{KEYWORDS}

Microwave NDE, near-field reflection method, bond quality inspection, defect detection and swept frequency NDE technique

\section{INTRODUCTION}

Fiber reinforced polymer (FRP) composites are employed in various applications of aerospace and defence industry. FRP composites are preferred as major structural parts due to their high stiffness strength and light weight. Also, their functional properties, namely mechanical, thermal and electrical can be tailor made to suit an application. FRP composite structures develop defects or damage during the fabrication process and in-service. Assessing the quality and health monitoring of the composite structure is of primary concern for meeting the intended application of the structure.Non-destructive evaluation (NDE) plays an important role in characterizing and confirming the quality of the product, for its functionality and applications. Ultrasonics, Thermography, X-ray radiography are the most common NDE techniques for evaluating the FRP composite structures [1]. Depending on the type of material, their characteristic features and accessibility of the test object, suitable techniques are employed.Microwave Non-destructive evaluation (MWNDE) is an emerging NDE technique for characterizing and inspecting the dielectric structures [2]. Microwave NDE operates in the frequency range $1 \mathrm{GHz}-40 \mathrm{GHz}$ of the electromagnetic spectrum. The following features of microwaves makes it a suitable and challenging NDE tool over the conventional NDE techniques: 
- Ability to penetrate dielectric materials

- No couplant required during testing

- Testing done in either near-field or far-field of the probe

- Single sided (Reflection method) or two sided (Transmission reflection method) inspection

- Provides contact mode or non-contact mode of testing

- Cost effective inspecting setup

Areas of application of microwave NDE include dielectric material characterization, monitoring or determining thickness variation, defect detection and bond quality inspection. Inspection of profiled or contoured FRP composite structures by near-field reflection microwave NDE technique is presented in this paper. Bond quality inspection of FRP composite structures with insulation rubber lining and thickness variation of composite structures respectively, are considered to establish Microwave NDE technique in both Contact and Non-contact modes respectively. Results of inspected profiled composite structures by swept frequency reflection microwave NDE technique in the X-band and Ku-band frequencies, respectively are presented.

\section{THEORETICAL BACKGROUND}

Any NDE technique is based on the interaction of the interrogating signal with a compatible material, which provides the required information about the material or structure [1]. In microwave NDE, the ability of microwaves to penetrate dielectric materials aids in evaluating dielectric FRP composite structures. A dielectric material is defined as a non-conductor of electricity which stores charge from an external electric field. A dielectric material is characterized by dielectric properties namely, dielectric constant and loss tangent at a particular frequency. When microwaves are incident on a dielectric material, part of the energy is reflected, part is transmitted through the material and remaining is absorbed. The distribution of energy into these three categories is due to the variation in the dielectric properties of the material from that of the external medium. The interaction of microwaves with the dielectric material in the form of transmission, reflection and absorption is the basis for microwave NDE $[3,4]$.

Near-field reflection microwave NDE is employed for inspection of dielectric FRP composites. Further, testing can be done in either contact or non-contact modes.A dielectric material/structure backed by conductor shown in Figure 1 is the testing configuration, where reflection coefficient, $S_{11}$ is the measurable parameter [2].Reflection coefficient consists of magnitude and phase information as a function of operating/testing frequency. For a material with low dielectric properties, a material backed by conductor configuration is used to enhance the reflected signal. As the test configuration involves conductor backup, this leads to singlesided inspection of the structure. Figure 2 shows the standard X-band (8.2-12.4GHz) Openended rectangular waveguide (OEWG) probe operating in dominant $\mathrm{TE}_{10}$ mode. For near field measurement, a square flange with sides greater than one-wavelength in free space is a good approximation to the theoretical assumption of an infinite flange where fields exists within the radiating aperture [2]. To enhance the detection sensitivity, operating frequency incase of both contact and non-contact methods, and stand-off distance incase of non-contact method is optimized. 
International Journal Of Microwave Engineering (JMICRO) Vol.5, No.1, January 2020

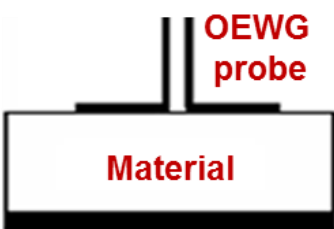

Conductor backup

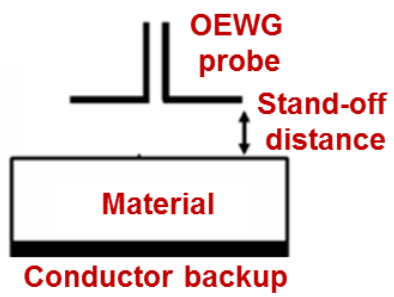

(b)Non contact method

(a) Contact method

Figure 1. Near-field reflection microwave NDE test configuration

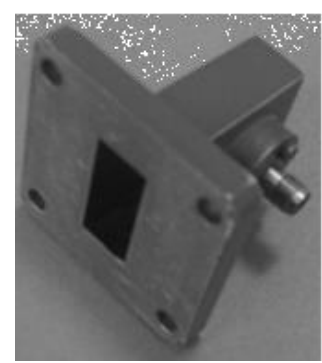

Figure 2. X-band (8.2-12.4GHz) Open-ended rectangular waveguide (OEWG) probe

\section{EXPERIMENT AND RESULTS}

The experimental setup for Near-field reflection microwave NDE in non-contact mode is shown in Figure 3. The setup consists of KeysightField Fox Microwave Vector Network Analyzer (VNA), coaxial cables, open-ended rectangular waveguide probe, waveguide calibration kit and sample under test backed by conductor [5]. Microwave VNA is a portable VNA with similar features of a bench top VNA and suitable for field or on-site applications. The experimental setup is calibrated for the test frequency and the reflection coefficient of a conductor is measured. The reflection coefficient of a conductor consists of both magnitude and phase, which is the reference. During the inspection, the test frequency is swept and hence the technique is also called as Swept frequency reflection microwave NDE technique

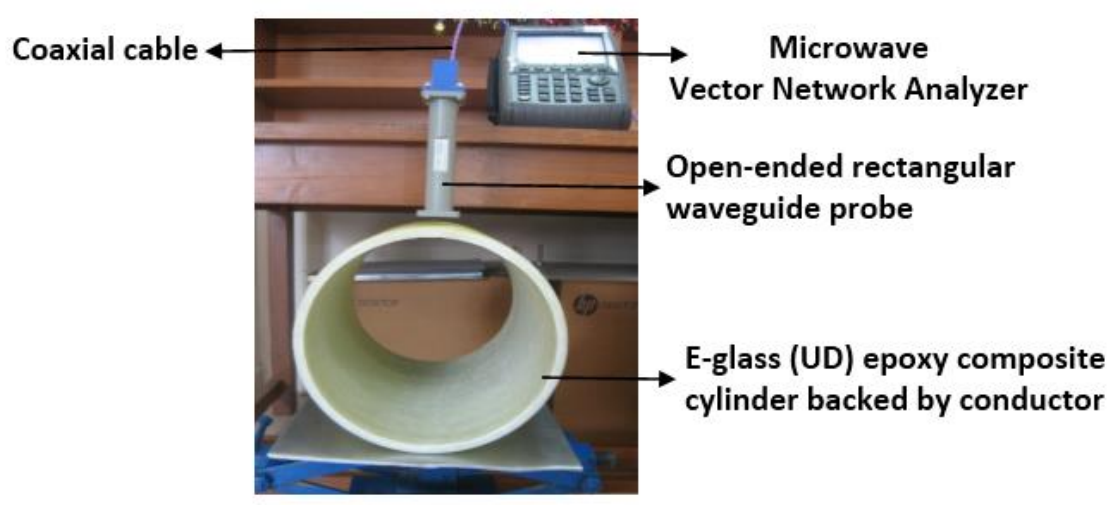

Figure 3. Near-field reflection microwave NDE in non-contact mode at X-band

To understand the interaction of microwaves with dielectric composites and establish the NDE technique,FRP composite laminates (flat structures) with known inserted defects are considered. 
International Journal Of Microwave Engineering (JMICRO) Vol.5, No.1, January 2020

Microwave NDE technique is established for defect detection, bond quality inspection and monitoring thickness variation [5, 6, 7].Inspection of FRP composite laminatesfor each of the mentioned areas, is carried out by measuring the reflection characteristics with respect to the

test frequency, in both contact and non-contact modes respectively. For a given material dielectric properties and thickness, the sensitivity to detect the defects depends on the response of the material to the frequency.Depending on the detection sensitivity, either magnitude or phase characteristics is an indicative parameter of the presence of defect (void, inclusion, debond, thickness variation, etc).Swept frequency reflection microwave NDE technique is automated to provide microwave $\mathrm{C}$-scan image of the inspected FRP composite laminate structures.

The established Microwave NDE technique on flat FRP composite structures (laminate level) is extended to profiled or contoured FRP composite structures. To establish the technique, the following profiled FRP composite structures are considered: (a) FRP composite cylinder, (b) FRP composite cylinder with insulation rubber lining, (c) composite pressure vessel (CPV) with insulation rubber lining and (d)radome.Inspection of each of the structures is carried out manually by measuring the reflection characteristics with respect to test frequency, in both contact and non-contact modes, respectively.

\subsection{Inspection of Uni-Directional Frp Composite Cylinder}

Uni-directional (UD) E-glass epoxy composite cylinder shown in Figure 3 is inspected by Microwave NDE non-contact technique [8]. The reflection characteristics of the composite cylinder is measured with respect to the fiber orientation, with the inspecting probe. The openended rectangular waveguide probe has electric field distribution perpendicular to the broader dimension ' $\mathrm{a}$ ' of the probe. The interaction of this electric field with the fiber orientation is studied. Two regions (A1 and A2, A2>A1) of the cylinder are inspected for the orientation of the probe with the fiber direction by non-contact method. The standoff distance is varied from $0 \mathrm{~mm}$ (contact) to $5 \mathrm{~mm}$ and the reflection characteristics are measured in the frequency range of $\mathrm{X}$-band frequency range.The results of reflection magnitude and reflection phase of the inspected composite cylinder at X-band are shown in Figure 4 and Figure 5, respectively for a standoff distance of $2 \mathrm{~mm}$.

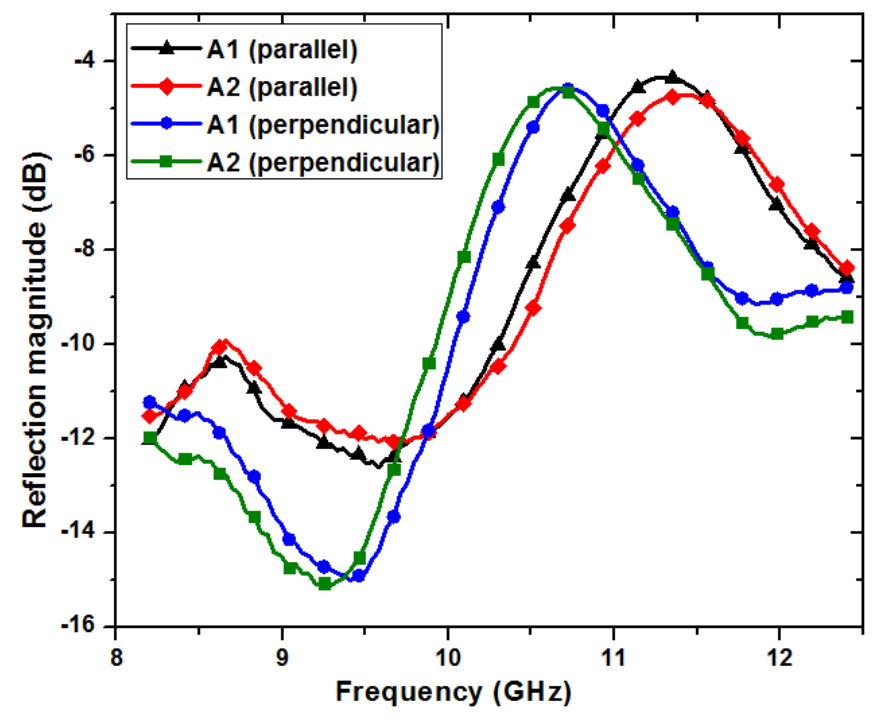

Figure 4.Reflection magnitude of E-glass (UD) epoxy composite cylinder with respect to fiber orientation with the probe at X-band 


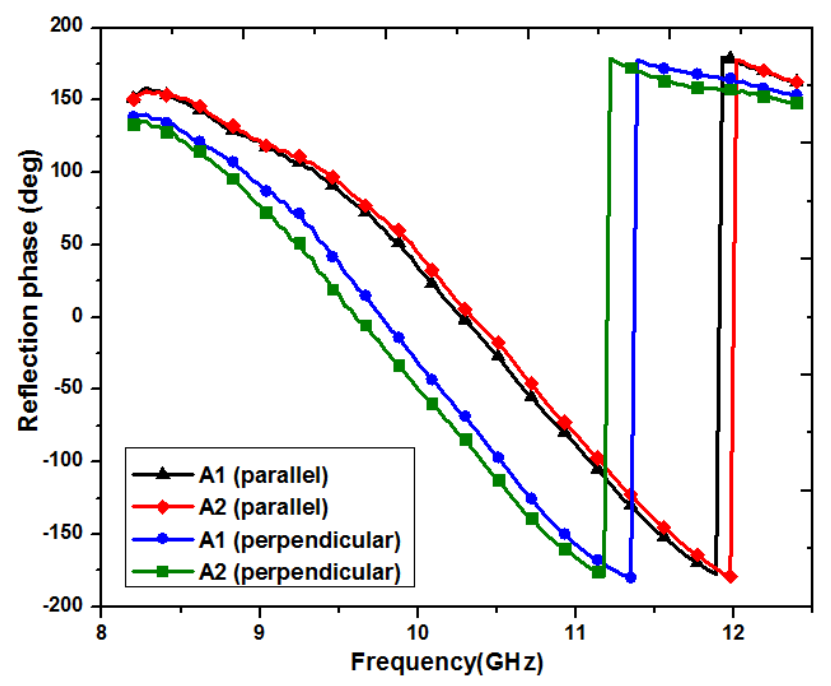

Figure 5. Reflection phase of E-glass (UD) epoxy composite cylinder with respect to fiber orientation with the probe at X-band

Inspection of the composite cylinder is carried out on the outer surface of the cylinder with conductor ( $\mathrm{Al}$ sheet) placed inside due to the probe orientation with the fiber direction. The reflection characteristics are measured for the two regions, by orienting the probe broader dimension ' $a$ ' parallel and perpendicular to the fiber direction, respectively. With respect to the probe orientation, both resulted in similar characteristics except with a shift of the characteristic curve to lower frequency in case of probe being perpendicular to the fiber direction. When the probe is perpendicular to the fiber direction, the electric field interacts with reinforcement (fiber) of higher dielectric constant, leading to the shift of the curve to the lower frequency side. In both, parallel and perpendicular orientations of the probe with the fiber direction, thickness variation of the inspected regions (A1 and A2) are observed. Thickness of region A2 is slightly higher than the region $\mathrm{A} 1$ by around $0.1 \mathrm{~mm}$ due to the fiber winding process. This thickness variation reflects in both the magnitude and phase curves obtained for an optimized standoff distance of $2 \mathrm{~mm}$.In parallel case, with increase in thickness, the characteristic curve shifts to higher frequency. In perpendicular case, with increase in thickness, the characteristic curve shifts to lower frequency side. The interaction of electric field with the volume of the fiber and matrix at the inspected region affects the position of the characteristic curve in both the cases.

\subsection{Inspection Of Uni-Directional Frp Composite Cylinder With InsulationR Rubber}

A uni-directional (UD) E-glass epoxy composite cylinder with an insulating rubber liningis shown in Figure 6inspected by the Microwave NDE non-contact technique [8]. The Glass epoxy composite cylinder is lined with an insulation rocasin rubber lining. The bond quality between the composite cylinder and the insulation rubber is inspected by measuring the reflection characteristics of the structure. Inspection is done from rubber side with conductor (Al plate) on outer side of the composite cylinder and inspecting probe broader dimension ' $a$ ' being parallel to the fiber direction. 
International Journal Of Microwave Engineering (JMICRO) Vol.5, No.1, January 2020

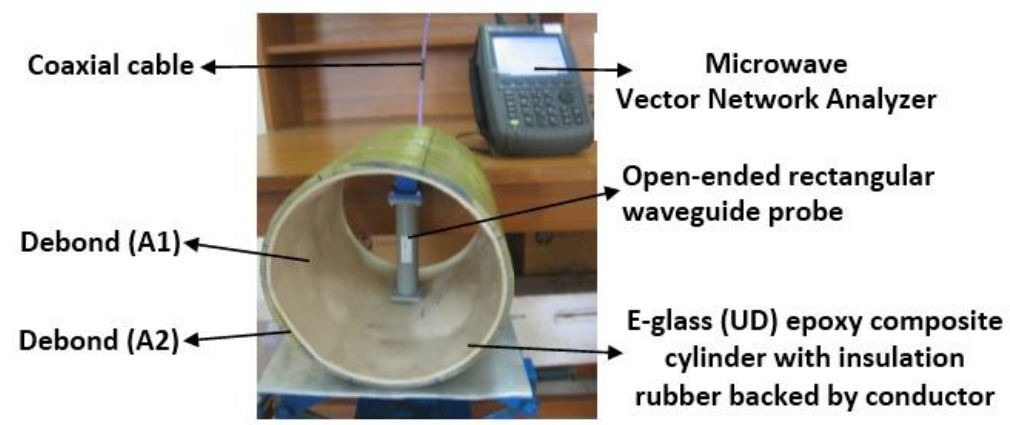

Figure 6. Near-field reflection microwave NDE of FRP composite cylinder with insulation rubber in non-contact mode at X-band

The results of reflection magnitude and reflection phase of the inspected composite cylinder with insulation rubber lining at X-bandfrequency range are shown in Figure 7 and Figure 8, respectively for anoptimized standoff distance of $2 \mathrm{~mm}$. Bond quality inspection of the entire structure is carried out which resulted in identifying many debond regions. Only two debond regions (A1 and A2, A2>A1) are reported in this paper.

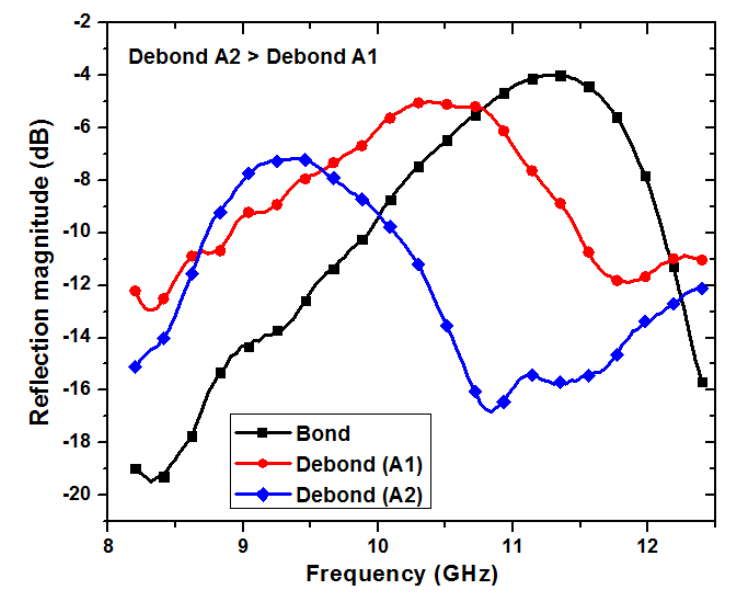

Figure 7. Reflection magnitude of E-glass (UD) epoxy composite cylinder with insulation rubber at $\mathrm{X}$-band

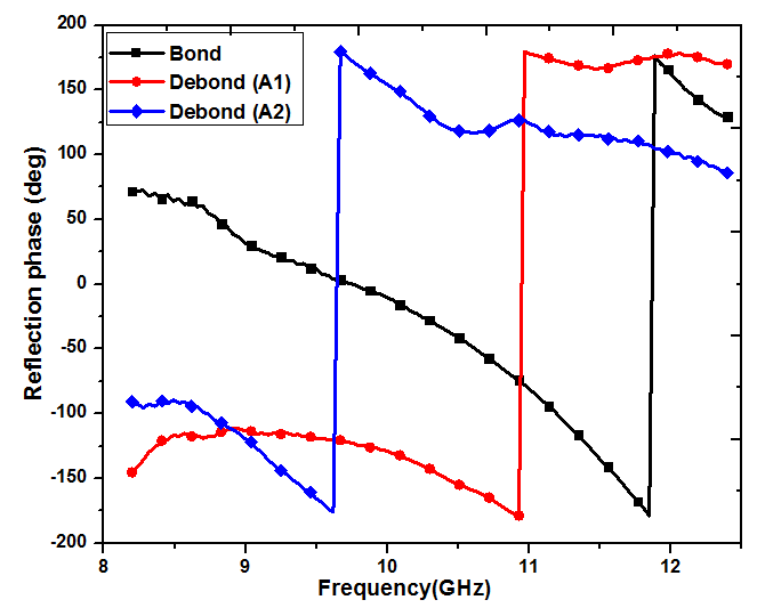

Figure 8. Reflection phase of E-glass (UD) epoxy composite cylinder with insulation rubber at $\mathrm{X}$-band 
The bond and debond regions are clearly distinguishable from the results of reflection magnitude and reflection phase of the inspected structure respectively. The sample configuration for bond region is (UD) E-glass epoxy composite bonded to rocasin rubber. For debond region, the configuration is (UD) E-glass epoxy composite and rocasin rubber separated by an air-gap, resulting in a change in the effective dielectric properties as compared to that of the bond region. Due to this, the debond curve shifts to lower frequency as compared to the bond curve.Also, with an increase in the debond thickness, the characteristic curve shifts to further lower frequency with respect to the bond curve. The resonance of the characteristic curve will indicate the debond thickness and hence can provide a quantitative measure of the debond thickness. A similar trend was observed from the results of bond quality inspection of flat structures (laminate level) inspected by the non-contact NDE technique [6]. From the magnitude characteristics, the resonance of the bond region occurs at higher frequency as compared withdebond regions. See data of debond (A1) and debond (A2) shown in the Figure 7 above.From the phase characteristics, it can been seen that the resonance of the bond region occurs at higher frequency as compared to debond regions (seeA1 and A2 data shown in figure 8). From the results it is observed that, phase characteristics provide much better representation (or indication) of the bond quality of the inspected structure.

\subsection{Inspection Of Composite Pressure Vessel With Insulation Rubber}

A composite pressure vessel (CPV) is an essential component of an aerospace structure. Fibre reinforced polymer (FRP) composite or metal forms are being used for the special applications. The bond quality of a pressure vessel and an insulation layer determines the aerospace mission objectives. Inspection of composite pressure vessel (CPV) model (shown in Figure 9), is carried out in the frequency range of $12.4-18 \mathrm{GHz}$ (Ku-band) by the contact method [6]. A customized fixture for holding the Open-ended rectangular waveguide probe is used for the inspection as shown in Figure 10.

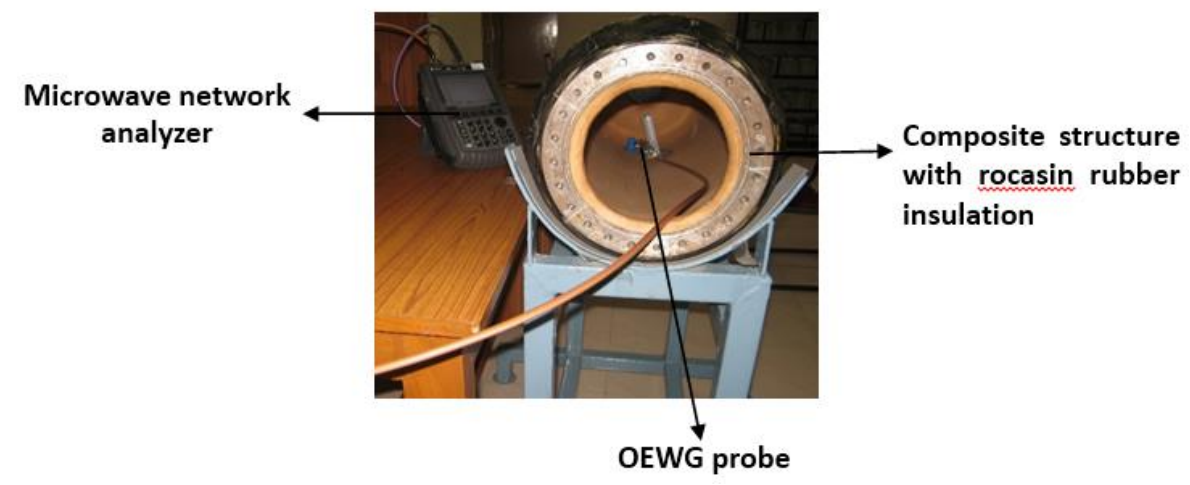

Figure 9. Experimental setup for inspecting composite pressure vessel withrocasin rubber insulation by contact method at $\mathrm{Ku}$-band

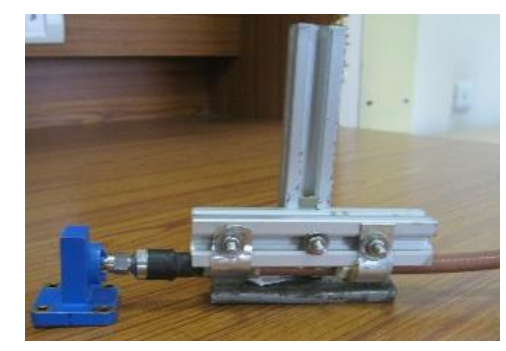

Figure 10. Customized fixture for holding OEWG probe 
Composite structure referred here for inspection consists of carbon epoxy composite with rocasin rubber as insulation layer. The composite casing structure is fabricated by unidirectional carbon epoxy (CE) material having both helical and hoop filament winding method. Because of the orientation of carbon epoxy material in the fabricated structure, the penetration of microwaves is limited. This makes carbon epoxy composite casing to have similar reflection characteristics as that of a conductor.

The magnitude and phase reflection characteristics of composite pressure vessel (CPV) with rocasin rubber insulation are shown in Figure 11 and 12 respectively. Carbon epoxy (CE) composite acts like a conductor backup and is taken as a reference. Results pertaining to two non-defect (bond) regions and two defect (debond) regions are reported. Characteristic curves A3 and A4 correspond to bond regions respectively. Characteristic curves A1 and A2 correspond to debond regions respectively where debond thickness of A1 is greater than that of A2. From the results, the bond and debond regions are distinguishable. From the reflection phase results of debond characteristics, the position of the curve indicates the debond thickness.

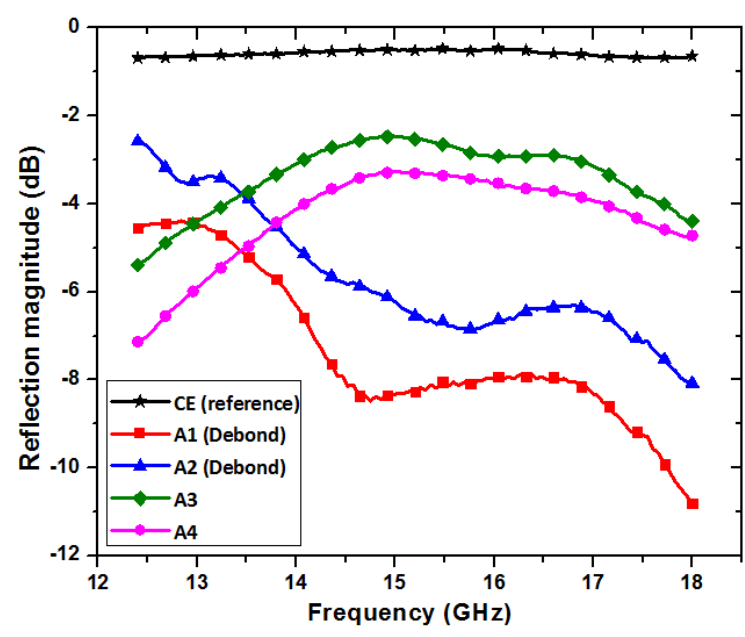

Figure 11. Reflection magnitude of CPV at Ku-band

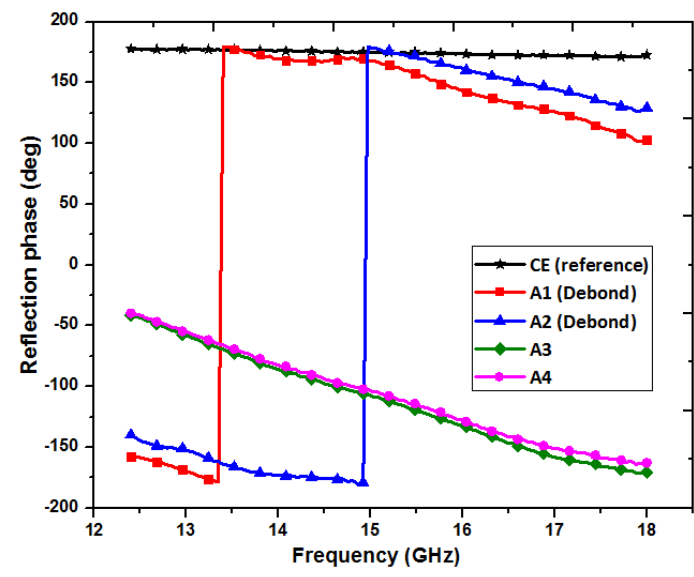

Figure 12. Reflection phase of CPV at Ku-band 
International Journal Of Microwave Engineering (JMICRO) Vol.5, No.1, January 2020

The bond quality of the inspected CPV is sensitive in the frequency range of $14-18 \mathrm{GHz}$ from the reflection magnitude characteristics and $12-18 \mathrm{GHz}$ from the reflection phase characteristics respectively.

\subsection{Inspection of Frp Composite Radome}

An FRP composite radome made of Quartz cyanate ester (QCE), shown in Figure 13 was inspected by Microwave NDE non-contact technique. The radome is fabricated by match die molding using bi-directional prepreg QCE socks. To establish Microwave NDE on the profiled structure and also study the effect of curvature on the inspected results, the radome structure is divided into regions namely, region $\mathrm{A}$, region $\mathrm{B}$ and region $\mathrm{C}$ respectively, along the radome circumference based on the surface curvature. Further each region is divided into 5 sub-regions namely, A1, A2, A3, A4 and A5 respectively related to region A and so on. The schematic for inspecting the radome is shown in Figure 14.

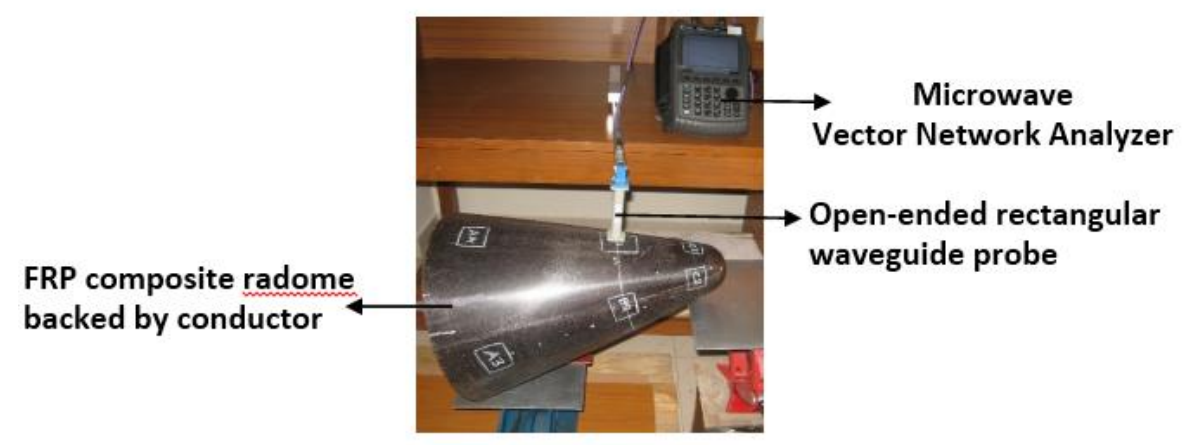

Figure 13. Experimental setup for inspecting composite radome by non-contact method at Ku-band

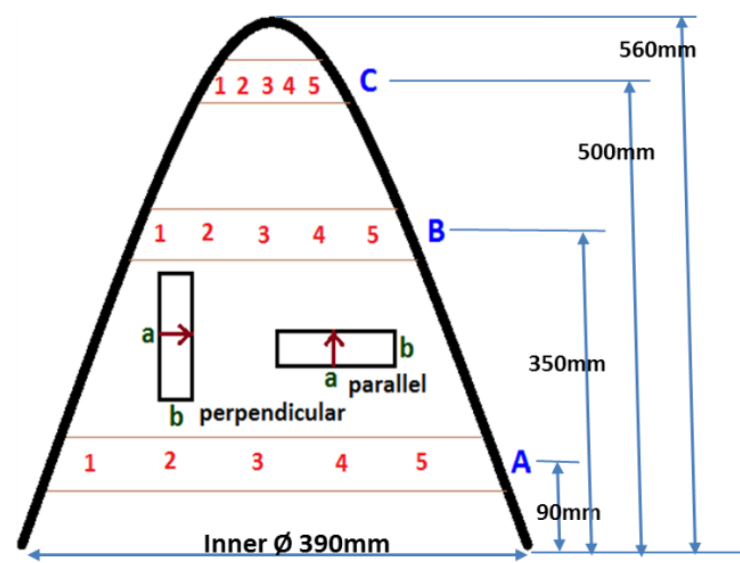

Figure 14. Schematic of inspection regions of radome

To study the effect of radome curvature, inspection of the structure is carried out in two conditions: by positioning the probe's broader dimension ' $a$ ', parallel and perpendicular to the composite surface respectively. The radome is inspected from outside with conductor backup (Al sheet) inside the structure. The reflection characteristics of each region is measured by noncontact technique in the frequency range of Ku-band for each of the test conditions under the assumption that the entire radome has uniform dielectric properties. Thickness variation across the inspected regions were observed, both from microwave NDE results and manual thickness measurement.Average thickness measured by Magnamikein the inspected regions are shown in Table 1.From the results, it was observed that reflection characteristics (magnitude and phase) 
International Journal Of Microwave Engineering (JMICRO) Vol.5, No.1, January 2020

obtained for perpendicular case followed a certain trendsimilar to as observed in flat structure (laminate level). The reason could be that the radome curvature effect is minimum when positioning the probe is perpendicular to the radome surface.

Table 1. Average thickness of inspection regions of a radome

\begin{tabular}{|c|c|c|c|c|c|}
\hline \multicolumn{6}{|c|}{ Average thickness (mm) $\pm 0.001 \mathrm{~mm}$} \\
\hline \multicolumn{2}{|c|}{ Region A } & \multicolumn{2}{c|}{ Region B } & \multicolumn{2}{c|}{ Region C } \\
\hline A1 & 4.91 & B1 & 5.08 & C1 & 4.73 \\
\hline A2 & 4.87 & B2 & 4.76 & C2 & 4.58 \\
\hline A3 & 4.81 & B3 & 5.04 & C3 & 4.68 \\
\hline A4 & 4.71 & B4 & 4.68 & C4 & 4.83 \\
\hline A5 & 4.80 & B5 & 4.94 & C5 & 5.17 \\
\hline
\end{tabular}

Results pertaining to the perpendicular case is reported in this paper for region $\mathrm{A}$ and region $\mathrm{C}$ respectively (region $\mathrm{C}$ has larger curvature than region $\mathrm{A}$ ). The results of reflection magnitude and reflection phase of the inspected composite radomefor region A (sub-regions: A1, A2, A3, A4 and A5) at Ku-band are shown in Figure 15 and Figure 16 respectively for an optimized standoff distance of $1 \mathrm{~mm}$.

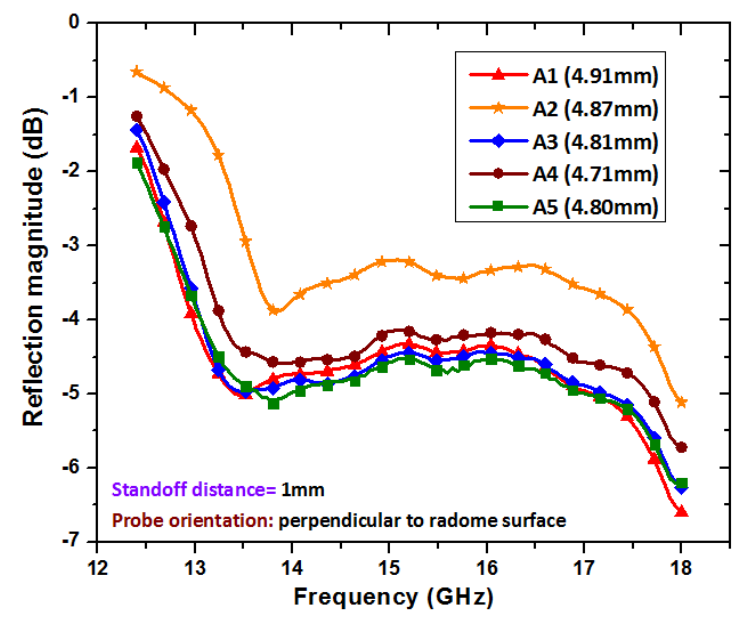

Figure 15. Reflection magnitude of QCE radome(region A) at Ku-band 
International Journal Of Microwave Engineering (JMICRO) Vol.5, No.1, January 2020

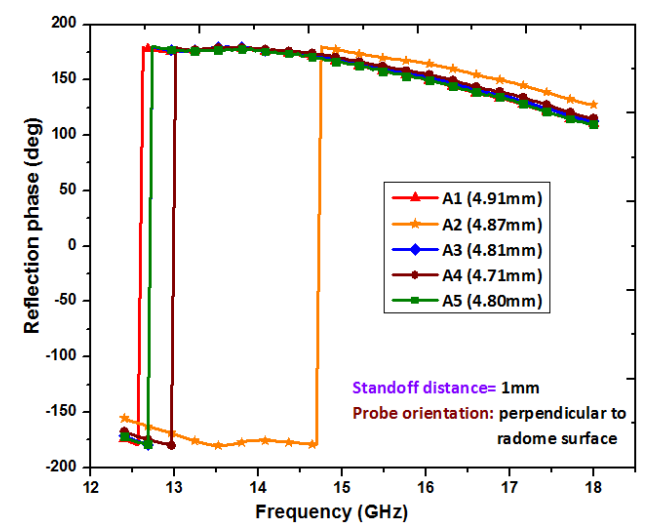

Figure 16. Reflection phase of QCE radome(region A) at Ku-band

Thickness variation in the inspected sub-regions of region A, of the composite radome clearly reflects in both the magnitude and phase characteristic curves. Thickness variation of $0.1 \mathrm{~mm}$ is observed in the curves for the inspected radome.From the results, it is observed that with increase in thickness, the characteristic curve shifts to the lower frequency. A similar trend was observed from the results of the flat structure (laminate level) inspected by non-contact NDE technique. The resonance of the characteristic curve depends on the response of the material with certain dielectric properties and thickness, with respect to the frequency. Deviation of the curve $\mathrm{A} 2$ from the trend can be attributed to variation in the dielectric properties at that region. The results of reflection magnitude and reflection phase of the inspected composite radome for region C (sub-regions: C1, C2, C3, C4 and C5) at Ku-band are shown in Figure 17 and Figure 18 respectively for an optimized standoff distance of $1 \mathrm{~mm}$. Region $\mathrm{C}$ has larger curvature as compared to region A. From the results, it is observed that with increase in the thickness, the characteristic curve shifts to the lower frequency.

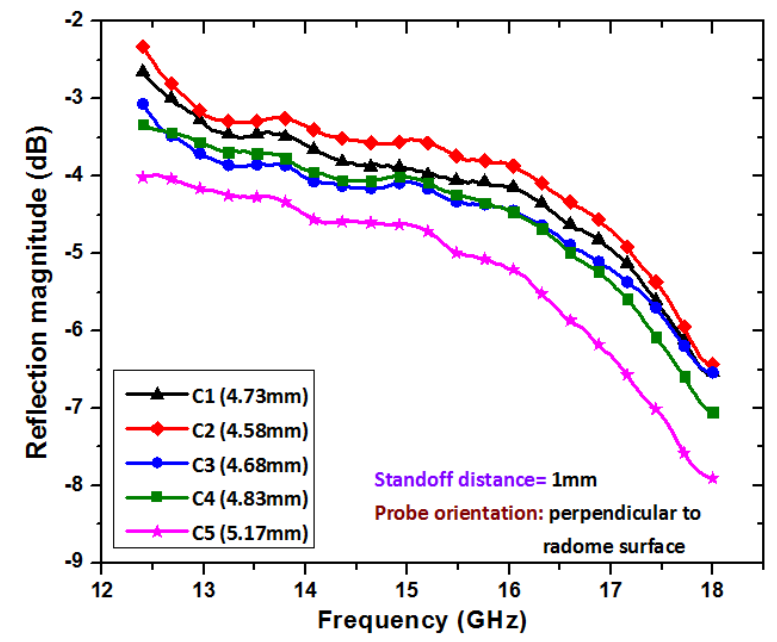

Figure 17. Reflection magnitude of QCE radome(region C) at Ku-band 
International Journal Of Microwave Engineering (JMICRO) Vol.5, No.1, January 2020

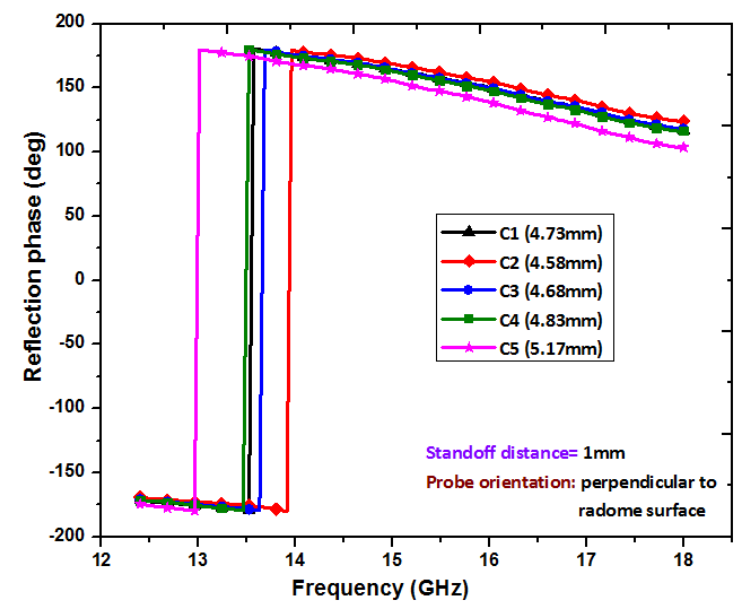

Figure 18. Reflection phase of QCE radome(region $C$ ) at Ku-band

FRP composite QCE radome was inspected by the thermography technique and the results did not detect these thickness variations. Based on the results of the inspected QCE radome by microwave NDE non-contact technique, the following observations are arrived at:

- By positioning the probe's broader dimension ' $a$ ' perpendicular to the surface of the inspected FRP composite radome, a certain trend in the reflection characteristics (magnitude and phase) is followed while inspecting for thickness variation in the structure.

- The observed trend is similar to the results obtained by inspecting the flat structure (laminate). So, the curvature has minimum effect on the measured characteristics in the perpendicular case.

- Any variation in thickness or dielectric properties clearly reflect in the characteristic curves.

- The resonance of the characteristic curve is an indicative parameter of the thickness and can provide a quantitative measure of the thickness.

\section{Conclusions}

Microwave non-destructive evaluation (MWNDE) is an emerging NDE technique for characterizing and inspecting dielectric composite structures. Microwave NDE non-contact technique is established on flat composite structures and extended its applicability to inspect profiled structures. The potential of Swept frequency reflection microwave NDE technique in inspecting various profiled composite structures for bond quality and thickness variation is observed. In the inspection of profiled composite structures, the response and position of the characteristic curve is an indicative parameter of the defect (debond, thickness). For low loss dielectric FRP composites, reflection phase is sensitive to the presence of defect. The technique can be automated to inspect profiled or curved dielectric structures for various applications such as thickness monitoring or measurement, defect detection and bond quality inspection.

\section{ACKNOWLEDGEMENTS}

The authors are thankful to the Director, Advanced Systems Laboratory (ASL) for providing an opportunity, support and encouragement to carry out the work. 
International Journal Of Microwave Engineering (JMICRO) Vol.5, No.1, January 2020

\section{REFERENCES}

[1] Peter. J. Shull, Nondestructive Evaluation- Theory, Techniques and Applications, Marcel Dekker, Inc. New York, 2002.

[2] Reza Zoughi, Microwave Non-Destructive Testing and Evaluation, Non-destructive evaluation series volume 4, Kluwer Academic Publishers, 2000.

[3] N. Ida, Microwave NDT, Developments in Electromagnetic theory and applications, volume 10, Kluwer Academic Publishers, 1992.

[4] David. M. Pozar, Microwave Engineering, Second edition, John Wiley \& Sons, Inc, 1998.

[5] Asha Gokul, K. Srinivas, D. Radhika and J. Dhanasekaran, Application of Microwave NDE for Dielectric FRP Composites, International Conference on Composite Materials and StructuresICCMS 2017.

[6] Asha Gokul, K. Srinivas and J. Dhanasekaran, Bond quality inspection of bonded surfaces using Microwave NDE, International Journal of Advances in Microwave Technology (IJAMT), Vol.4, No.4, November 2019.

[7] Asha Gokul, K. Srinivas, D. Radhika and J. Dhanasekaran, Inspection of GFRP composites by Microwave NDE, accepted for publication in International Journal of Microwave Engineering (JMICRO).

[8] S. K. Sahoo, Asha Gokul, K. Srinivas, R. N. Rao and M. K. Buragohain, Comparison of NMR and Microwave NDE methods for defect detection in composite structures, Materials Today: Proceedings, Volume 18, Part 7, 2019, Pages 4074-4081.

\section{AUTHORS}

Asha Gokul received Bachelor of Engineering (B. E) degree in Electronics and Communication Engineering and Masters of Engineering (M. E) degree in Microwave and Radar Engineering from Osmania University, Hyderabad, India. She is currently working as Scientist in Advanced Systems Laboratory, DRDO, Hyderabad. Her areas of work include Electromagnetic design of radomes, Dielectric material characterization and Microwave NDE.She has published international and conference papers.

Srinivas Kuchipudireceived PhD degree in Physics from Osmania University, Hyderababd, India. He is currently working as Scientist in Advanced Systems Laboratory, DRDO, Hyderabad. His areas of work include NDE of Composite structures and characterization of composites, ferroelectrics, microwave NDE techniques. He has published over 28 international publications and conference

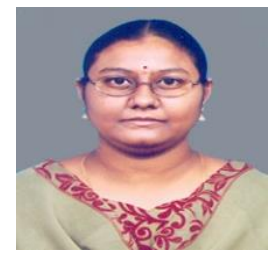
papers.

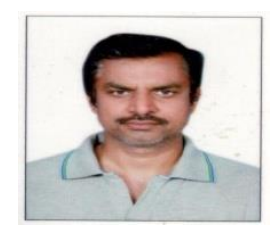

